PROCEEDINGS OF THE

AMERICAN MATHEMATICAL SOCIETY

Volume 130, Number 12, Pages 3631-3640

S 0002-9939(02)06615-7

Article electronically published on May 14, 2002

\title{
HAHN-BANACH EXTENSION OPERATORS AND SPACES OF OPERATORS
}

\author{
ÅSVALD LIMA AND EVE OJA
}

(Communicated by N. Tomczak-Jaegermann)

\begin{abstract}
Let $X \subseteq Y$ be Banach spaces and let $\mathcal{A} \subseteq \mathcal{B}$ be closed operator ideals. Let $Z$ be a Banach space having the Radon-Nikodým property. The main results are as follows. If $\Phi: \mathcal{A}(Z, X)^{*} \rightarrow \mathcal{B}(Z, Y)^{*}$ is a Hahn-Banach extension operator, then there exists a set of Hahn-Banach extension operators $\phi_{i}: X^{*} \rightarrow Y^{*}, i \in I$, such that $Z=\sum_{i \in I} \oplus_{1} Z_{\Phi \phi_{i}}$, where $Z_{\Phi \phi_{i}}=\{z \in$ $\left.Z: \Phi\left(x^{*} \otimes z\right)=\left(\phi_{i} x^{*}\right) \otimes z, \forall x^{*} \in X^{*}\right\}$. If $\mathcal{A}(\hat{Z}, X)$ is an ideal in $\mathcal{B}(\hat{Z}, Y)$ for all equivalently renormed versions $\hat{Z}$ of $Z$, then there exist Hahn-Banach extension operators $\Phi: \mathcal{A}(Z, X)^{*} \rightarrow \mathcal{B}(Z, Y)^{*}$ and $\phi: X^{*} \rightarrow Y^{*}$ such that $Z=Z_{\Phi \phi}$.
\end{abstract}

\section{INTRODUCTION}

Let $X$ be a closed subspace of a Banach space $Y$. A linear operator $\phi: X^{*} \rightarrow Y^{*}$ is called a Hahn-Banach extension operator if $\left(\phi x^{*}\right)(x)=x^{*}(x)$ and $\left\|\phi x^{*}\right\|=\left\|x^{*}\right\|$ for all $x \in X$ and all $x^{*} \in X^{*}$. Let us denote the set of all Hahn-Banach extension operators $\phi: X^{*} \rightarrow Y^{*}$ by $H B(X, Y)$.

It is well known (and straightforward to verify) that $H B(X, Y) \neq \emptyset$ if and only if $X$ is an ideal in $Y$. Let us recall that $X$ is an ideal in $Y$ if $X^{\perp}$, the annihilator of $X$ in $Y^{*}$, is the kernel of a norm one projection on $Y^{*}$. The notion of an ideal was introduced and studied by Godefroy, Kalton, and Saphar in [7].

For a Banach space $Z \neq\{0\}$, let $\mathcal{A}$ be a closed subspace of the Banach space $\mathcal{L}(Z, X)$ of bounded linear operators and let $\mathcal{B}$ be a closed subspace of $\mathcal{L}(Z, Y)$. We assume that the subspaces of finite rank operators $\mathcal{F}(Z, X)$ and $\mathcal{F}(Z, Y)$ are respectively contained in $\mathcal{A}$ and $\mathcal{B}$. We also assume that $\mathcal{A} \subseteq \mathcal{B}$.

If $\Phi \in H B(\mathcal{A}, \mathcal{B})$, then it is easy to see that any pair of elements $z \in Z, z^{*} \in Z^{*}$ satisfying $\|z\|=\left\|z^{*}\right\|=z^{*}(z)=1$ defines $\phi \in H B(X, Y)$ by

$$
\left(\phi x^{*}\right)(y)=\left(\Phi\left(x^{*} \otimes z\right)\right)\left(z^{*} \otimes y\right), \quad \forall x^{*} \in X^{*}, \forall y \in Y .
$$

Received by the editors July 16, 2001.

2000 Mathematics Subject Classification. Primary 46B20, 46B28, 47L05.

Key words and phrases. Hahn-Banach extension operators, spaces of operators, operator ideals, Radon-Nikodým property.

The second-named author wishes to acknowledge the warm hospitality provided by Åsvald Lima and his colleagues at Agder College, where a part of this work was done in October 2000. Her visit was supported by the Norwegian Academy of Science and Letters and by Estonian Science Foundation Grant 4400. 
This means that

$$
\left(\phi x^{*}\right)(T z)=\left(\Phi\left(x^{*} \otimes z\right)\right)(T)
$$

for all $x^{*} \in X^{*}$ and all elementary operators $T \in \mathcal{B}$ of the form $T=z^{*} \otimes y, y \in Y$.

For given $\Phi \in H B(\mathcal{A}, \mathcal{B})$ and $\phi \in H B(X, Y)$, let us introduce

$$
Z_{\Phi \phi}=\left\{z \in Z:\left(\phi x^{*}\right)(T z)=\left(\Phi\left(x^{*} \otimes z\right)\right)(T), \forall x^{*} \in X^{*}, \forall T \in \mathcal{B}\right\} .
$$

Then $Z_{\Phi \phi}$ is a closed subspace of $Z$ and we may have $Z_{\Phi \phi}=\{0\}$. One of the main results of 9 asserts that if $X^{*}$ has the compact approximation property with conjugate operators (i.e. there exists a net $\left(K_{\alpha}\right)$ in $\mathcal{K}(X, X)$ such that $\left(K_{\alpha}^{*}\right)$ converges to the identity $I_{X^{*}}$ uniformly on compact subsets of $\left.X^{*}\right)$, then for any reflexive Banach space $Z$ and any $\phi \in H B(X, Y)$ there exists $\Phi \in H B(\mathcal{K}(Z, X), \mathcal{W}(Z, Y))$ so that $Z_{\Phi \phi}=Z$.

In this paper, we shall look at the general situation: we shall not assume any approximation property. We shall prove (see Theorems 1.3 and 2.3) that if a Banach space $Z$ has the Radon-Nikodým property and does not contain any isometric copy of $\ell_{1}$, then for every $\Phi \in H B(\mathcal{A}, \mathcal{B})$ there exists a finite set $\left\{\phi_{1}, \ldots, \phi_{n}\right\} \subseteq$ $H B(X, Y)$ so that

$$
Z=Z_{\Phi \phi_{1}} \oplus_{1} \cdots \oplus_{1} Z_{\Phi \phi_{n}},
$$

and, under some additional hypotheses, there exist $\phi \in H B(X, Y)$ and $\Phi \in$ $H B(\mathcal{A}, \mathcal{B})$ so that $Z=Z_{\Phi \phi}$.

The notation we use is standard. We consider Banach spaces over the real field $\mathbb{R}$. The closure of a set $A$ is denoted by $\bar{A}$ and its convex hull by conv $A$. For a Banach space $Z$ and $r>0$, we denote $B_{Z}(0, r)=\{z \in Z:\|z\| \leq r\}$, $S_{Z}(0, r)=\{z \in Z:\|z\|=r\}$, and $B_{Z}=B_{Z}(0,1)$. The set of denting points of $B_{Z}(0, r)$ is denoted by dent $B_{Z}(0, r)$. The identity operator on $Z$ is denoted by $I_{Z}$. By $\overline{\mathcal{F}(Z, Y)}, \mathcal{K}(Z, Y)$, and $\mathcal{W}(Z, Y)$ we denote the subspaces of $\mathcal{L}(Z, Y)$ of approximable operators (i.e. norm limits of finite rank operators), compact operators, and weakly compact operators.

\section{Hahn-BANACH EXTENSION OPERATORS AND SPACES OF OPERATORS}

Let $X$ be a closed subspace of a Banach space $Y$ and let $Z$ be a Banach space. Let $\mathcal{A} \subseteq \mathcal{L}(Z, X)$ and $\mathcal{B} \subseteq \mathcal{L}(Z, Y)$ be closed subspaces such that $\mathcal{F}(Z, X) \subseteq \mathcal{A}$, $\mathcal{F}(Z, Y) \subseteq \mathcal{B}$, and $\mathcal{A} \subseteq \mathcal{B}$.

For given $\phi \in H B(X, Y)$ and $\Phi \in H B(\mathcal{A}, \mathcal{B})$, we have mappings

$$
\phi \otimes I_{Z}: X^{*} \otimes Z \rightarrow Y^{*} \otimes Z \subseteq \mathcal{B}^{*}
$$

and

$$
\Phi_{\mid X^{*} \otimes Z}: X^{*} \otimes Z \rightarrow \mathcal{B}^{*} .
$$

By definition, $Z_{\Phi \phi}$ is the maximal subspace of $Z$ for which

$$
\phi \otimes I_{Z_{\Phi \phi}}=\Phi_{\mid X^{*} \otimes Z_{\Phi \phi}} .
$$

Thus

$$
Z_{\Phi \phi}=\left\{z \in Z: \Phi\left(x^{*} \otimes z\right)=\left(\phi x^{*}\right) \otimes z, \forall x^{*} \in X^{*}\right\} .
$$


Lemma 1.1. Let $X$ be a closed subspace of a Banach space $Y$ and let $Z$ be a Banach space. Let $\mathcal{A} \subseteq \mathcal{L}(Z, X)$ and $\mathcal{B} \subseteq \mathcal{L}(Z, Y)$ be closed subspaces such that $\mathcal{F}(Z, X) \subseteq \mathcal{A}, \mathcal{F}(Z, Y) \subseteq \mathcal{B}$, and $\mathcal{A} \subseteq \mathcal{B}$. Assume that $\Phi \in H B(\mathcal{A}, \mathcal{B})$. If $\phi_{1}, \phi_{2}, \ldots$ are in $H B(X, Y)$, all different, and if $z_{i} \in Z_{\Phi \phi_{i}}$ are such that $\sum_{i=1}^{\infty} z_{i}=0$ and $\sum_{i=1}^{\infty}\left\|z_{i}\right\|<\infty$, then $z_{i}=0$ for all $i \in \mathbb{N}$.

Proof. Assume for contradiction that some $z_{i}$, say $z_{1} \neq 0$. For $x^{*} \in X^{*}$ we get that

$$
0=\Phi\left(x^{*} \otimes 0\right)=\Phi\left(x^{*} \otimes \sum_{i=1}^{\infty} z_{i}\right)=\sum_{i=1}^{\infty} \Phi\left(x^{*} \otimes z_{i}\right)=\sum_{i=1}^{\infty}\left(\phi_{i} x^{*}\right) \otimes z_{i} .
$$

Let $z^{*} \in Z^{*}$ and $y \in Y$. Evaluating at $z^{*} \otimes y$ gives

$$
\begin{aligned}
0 & =\sum_{i=1}^{\infty}\left(\left(\phi_{i} x^{*}\right) \otimes z_{i}\right)\left(z^{*} \otimes y\right) \\
& =\sum_{i=1}^{\infty}\left(\phi_{i} x^{*}\right)(y) z^{*}\left(z_{i}\right) \\
& =z^{*}\left(\sum_{i=1}^{\infty}\left(\phi_{i} x^{*}\right)(y) z_{i}\right) .
\end{aligned}
$$

Since this is true for all $z^{*} \in Z^{*}$, we get $\sum_{i=1}^{\infty}\left(\phi_{i} x^{*}\right)(y) z_{i}=0$.

We can repeat this argument, and then we get

$$
\sum_{i=1}^{\infty}\left(\left(\phi_{i} x^{*}\right)(y)\right)^{m} z_{i}=0
$$

for all $m \in \mathbb{N}$ and all $x^{*} \in X^{*}, y \in Y$. But then, for every polynomial $p$ and all $x^{*} \in X^{*}, y \in Y$,

$$
\sum_{i=1}^{\infty} p\left(\left(\phi_{i} x^{*}\right)(y)\right) z_{i}=0
$$

Using the Weierstrass theorem, for every continuous function $f \in \mathcal{C}[-1,1]$ and all $x^{*} \in B_{X^{*}}, y \in B_{Y}$, we get

$$
\sum_{i=1}^{\infty} f\left(\left(\phi_{i} x^{*}\right)(y)\right) z_{i}=0
$$

Since the operators $\phi_{i}$ are all different, we can choose $x^{*} \in B_{X^{*}}$ and $y \in B_{Y}$ such that $\left(\phi_{1} x^{*}\right)(y) \neq\left(\phi_{2} x^{*}\right)(y)$. We now choose $f \in \mathcal{C}[-1,1]$ such that $f\left(\left(\phi_{1} x^{*}\right)(y)\right)=$ $1, f\left(\left(\phi_{2} x^{*}\right)(y)\right)=0$, and $\sup _{t \in[-1,1]}|f(t)|=1$. Then writing $a_{2 i}=f\left(\left(\phi_{i} x^{*}\right)(y)\right)$, we get $\left|a_{2 i}\right| \leq 1$ and

$$
z_{1}+\sum_{i=3}^{\infty} a_{2 i} z_{i}=0
$$

Note that $z_{1} \in Z_{\Phi \phi_{1}}, a_{2 i} z_{i} \in Z_{\Phi \phi_{i}}$, and $\left\|z_{1}\right\|+\sum_{i=3}^{\infty}\left\|a_{2 i} z_{i}\right\|<\infty$. Therefore, by the argument above, for all $f \in \mathcal{C}[-1,1]$ and all $x^{*} \in B_{X^{*}}, y \in B_{Y}$, we have

$$
f\left(\left(\phi_{1} x^{*}\right)(y)\right) z_{1}+\sum_{i=3}^{\infty} a_{2 i} f\left(\left(\phi_{i} x^{*}\right)(y)\right) z_{i}=0 .
$$


Consequently, we can find numbers $a_{j i} \in[-1,1]$ such that for every $m \in \mathbb{N}$

$$
z_{1}+\sum_{i=m+1}^{\infty} a_{2 i} \cdots a_{m i} z_{i}=0 .
$$

This implies that $\left\|z_{1}\right\| \leq \sum_{i=m+1}^{\infty}\left\|z_{i}\right\| \rightarrow 0$ when $m \rightarrow \infty$. Hence $z_{1}=0$, and we have a contradiction.

Lemma 1.2. Let $X, Y, Z, \mathcal{A}$, and $\mathcal{B}$ be as in Lemma 1.1. If $\Phi \in H B(\mathcal{A}, \mathcal{B})$ and $r>0$, then

$$
\operatorname{dent} B_{Z}(0, r) \subseteq S_{Z}(0, r) \cap \bigcup_{\phi \in H B(X, Y)} Z_{\Phi \phi} .
$$

Proof. Since dent $B_{Z}(0, r)=r \cdot \operatorname{dent} B_{Z}$ and $Z_{\Phi \phi}$ is a subspace, it suffices to consider dent $B_{Z}$. Let $z \in \operatorname{dent} B_{Z}$. The proof of [9, Lemma 3.1] shows that then, for every $x^{*} \in X^{*}$, all norm-preserving extensions of $x^{*} \otimes z \in \mathcal{F}(Z, X)^{*}$ to $\mathcal{L}(Z, Y)$ have the form $y^{*} \otimes z$, where $y^{*} \in Y^{*}$ is a norm-preserving extension of $x^{*}$. Recalling that $\Phi\left(x^{*} \otimes z\right) \in \mathcal{B}^{*}$ is a norm-preserving extension of $x^{*} \otimes z \in \mathcal{A}^{*}$ and using the linearity of $\Phi$, we see that the map $\phi: X^{*} \rightarrow Y^{*}$, which assigns to every $x^{*} \in X^{*}$ its norm-preserving extension $y^{*}=\phi x^{*} \in Y^{*}$ satisfying

$$
\Phi\left(x^{*} \otimes z\right)=\left(\phi x^{*}\right) \otimes z,
$$

is linear. Therefore $\phi \in H B(X, Y)$ and $z \in Z_{\Phi \phi}$.

Let $I$ be an arbitrary index set. Let $Z$ be a Banach space and let $\left(Z_{i}\right)_{i \in I}$ be a family of closed subspaces of $Z$. We shall call $Z$ an $\ell_{1}$-direct sum of subspaces $Z_{i}$, $i \in I$, and write

$$
Z=\sum_{i \in I} \oplus_{1} Z_{i}
$$

if every $z \in Z$ admits a decomposition $z=\sum_{i \in I} z_{i}$ with $z_{i} \in Z_{i}$ (meaning that the directed system of finite partial sums converges to $z$ ) such that $\|z\|=\sum_{i \in I}\left\|z_{i}\right\|$ and $0=\sum_{i \in I} z_{i}$ implies $z_{i}=0$ for all $i \in I$. Let us recall that the absolute summability of $\left(z_{i}\right)_{i \in I}$ (i.e. $\sum_{i \in I}\left\|z_{i}\right\|<\infty$ ) guarantees that the family $\left(z_{i}\right)_{i \in I}$ cannot contain more than countably many non-zero elements. This easily implies that every $z \in \sum_{i \in I} \oplus_{1} Z_{i}$ has, in fact, a unique representation $z=\sum_{i \in I} z_{i}$, where $z_{i} \in Z_{i}$. It is clear that if the index set is finite, then $Z$ is the $\ell_{1}$-direct sum in the usual sense: $Z=Z_{1} \oplus \ldots \oplus Z_{n}$ and $\|z\|=\sum_{i=1}^{n}\left\|z_{i}\right\|$ for all $z=\sum_{i=1}^{n} z_{i}$ in $Z$.

Theorem 1.3. Let $X, Y, Z, \mathcal{A}$, and $\mathcal{B}$ be as in Lemma 1.1. If $Z$ has the RadonNikodým property and $\Phi \in H B(\mathcal{A}, \mathcal{B})$, then there exists $\left\{\phi_{i}: i \in I\right\} \subseteq H B(X, Y)$ such that

$$
Z=\sum_{i \in I} \oplus_{1} Z_{\Phi \phi_{i}}
$$

If, moreover, $Z$ does not contain any isometrically isomorphic copy of $\ell_{1}$, then there exists a finite set $\left\{\phi_{1}, \ldots, \phi_{n}\right\} \subseteq H B(X, Y)$ such that

$$
Z=Z_{\Phi \phi_{1}} \oplus_{1} \cdots \oplus_{1} Z_{\Phi \phi_{n}} .
$$


Proof. Let $\left\{\phi \in H B(X, Y): Z_{\Phi \phi} \neq\{0\}\right\}=\left\{\phi_{i}: i \in I\right\}$ and let $W$ be the Cartesian $\ell_{1}$-product of the spaces $Z_{\Phi \phi_{i}}, i \in I$. Define an operator $U: W \rightarrow Z$ by $U\left(\left(z_{i}\right)_{i \in I}\right)=\sum_{i \in I} z_{i}$. Clearly $U$ is a continuous linear operator with $\|U\| \leq 1$. That $U$ is one-to-one follows from Lemma 1.1.

Recall that $B_{Z}(0, r)=\overline{\operatorname{conv}}$ dent $B_{Z}(0, r)$ for all $r>0$, because $Z$ has the RadonNikodým property.

Let $z \in Z, z \neq 0$, and let $\epsilon>0$. We can choose $u_{1} \in \operatorname{conv} \operatorname{dent} B_{Z}(0,\|z\|)$ with $\left\|z-u_{1}\right\| \leq \epsilon / 2$. Using Lemma 1.2 we can write

$$
u_{1}=\sum_{j=1}^{j(1)} \lambda_{j} z_{j}, \lambda_{j}>0, \sum_{j=1}^{j(1)} \lambda_{j}=1, z_{j} \in S_{Z}(0,\|z\|) \cap\left(\bigcup_{\phi \in H B(X, Y)} Z_{\Phi \phi}\right) .
$$

Next we choose $u_{2} \in$ conv $\operatorname{dent} B_{Z}(0, \epsilon / 2)$ with $\left\|z-u_{1}-u_{2}\right\| \leq \epsilon / 2^{2}$. Thus an induction procedure yields a sequence $u_{n} \in \operatorname{conv} \operatorname{dent} B_{Z}\left(0, \epsilon / 2^{n-1}\right)$ such that $z=$ $\sum_{n=1}^{\infty} u_{n}$. Let for $n \geq 2$

$$
\begin{aligned}
& u_{n}=\sum_{j=j(n-1)+1}^{j(n)} \lambda_{j} z_{j}, \lambda_{j}>0, \sum_{j=j(n-1)+1}^{j(n)} \lambda_{j}=1, \\
& z_{j} \in S_{Z}\left(0, \epsilon / 2^{n-1}\right) \cap\left(\bigcup_{\phi \in H B(X, Y)} Z_{\Phi \phi}\right) .
\end{aligned}
$$

Notice that $\sum_{j=1}^{\infty}\left\|\lambda_{j} z_{j}\right\| \leq\|z\|+\epsilon$ and $z=\sum_{j=1}^{\infty} \lambda_{j} z_{j}$. Therefore, by a rearrangement, we can write $z=\sum_{j=1}^{\infty} w_{j}$ with $w_{j} \in Z_{\Phi \phi_{j}}$ so that all $\phi_{j}$ are different. But then $U\left(\left(w_{j}\right)_{j=1}^{\infty}\right)=z$ and $\left\|\left(w_{j}\right)_{j=1}^{\infty}\right\|=\sum_{j=1}^{\infty}\left\|w_{j}\right\| \leq\|z\|+\epsilon$. Since $U$ is one-to-one and $\|U\| \leq 1$, this implies that $\left\|\left(w_{j}\right)_{j=1}^{\infty}\right\|=\|z\|$. Thus $U$ is a linear isometry from $W$ onto $Z$ and therefore

$$
Z=\sum_{i \in I} \oplus_{1} Z_{\Phi \phi_{i}}
$$

as desired.

For the "moreover" part, let us just note that if $I$ were infinite, then $Z$ should contain an isometrically isomorphic copy of $\ell_{1}$.

The Radon-Nikodým property is studied in detail e.g. in [6]. The RadonNikodým property for a dual space $W^{*}$ is equivalent to the requirement that separable subspaces of $W$ have separable duals. Thus all reflexive and all separable dual spaces have the Radon-Nikodým property .

By a well-known distortion result of James [8] (see e.g [10, p. 97]), if $Z$ contains a subspace isomorphic to $\ell_{1}$, then $Z$ contains "nearly isometric" copies of $\ell_{1}$. If $Z$ happens to be separable (e.g. $Z=\ell_{1}$ ), then, by a well-known result of Clarkson [4] (see e.g. [5, pp. 101 and 144]), $Z$ admits an equivalent strictly convex norm. The renormed version of $Z$ contains "nearly isometric" copies of $\ell_{1}$, but it does not contain any isometric copy of $\ell_{1}$.

Theorem 1.3 shows, in particular, that $Z=Z_{\Phi \phi_{1}} \oplus_{1} \cdots \oplus_{1} Z_{\Phi \phi_{n}}$ whenever $Z$ is a reflexive Banach space and $H B(\mathcal{A}, \mathcal{B}) \neq \emptyset$, where $\mathcal{A}$ and $\mathcal{B}$ are arbitrary operator spaces (satisfying some natural requirements like $\mathcal{F}(Z, X) \subseteq \mathcal{A}$ ). This is to compare with one of the main results in [9, Theorem 4.6] showing that under the hypothesis that $X^{*}$ has the compact approximation property with conjugate operators, for any reflexive Banach space $Z$, one has $H B(\mathcal{K}(Z, X), \mathcal{W}(Z, Y)) \neq \emptyset$ and $Z=Z_{\Phi \phi}$. 


\section{HAhN-BANACH EXTENSION OPERATORS AND SPACES OF OPERATORS GENERATED BY OPERATOR IDEALS}

Concerning the notation $\mathcal{A}$ and $\mathcal{B}$ from the previous section, it is convenient to change our notation as follows. In this section $\mathcal{A}$ and $\mathcal{B}$ will denote closed operator ideals in the sense of Pietsch [11. This guarantees, in particular, that $\mathcal{F}(Z, X) \subseteq$ $\mathcal{A}(Z, X)$ and $\mathcal{A}(Z, X)$ is a closed subspace of $\mathcal{L}(Z, X)$. This also guarantees that if $T \in \mathcal{A}(Z, X)$ and $U \in \mathcal{L}\left(Z_{0}, Z\right), V \in \mathcal{L}\left(X, X_{0}\right)$, then $V \circ T \circ U \in \mathcal{A}\left(Z_{0}, X_{0}\right)$. Let us recall that, for instance, the classes of all approximable operators, all compact operators, and all weakly compact operators are closed operator ideals.

Let us begin with an easy result which applies to arbitrary $Z$ and $\mathcal{A}$.

Proposition 2.1. Let $X$ be a closed subspace of a Banach space $Y$, let $Z$ be a Banach space and let $\mathcal{A}$ be a closed operator ideal. If $X$ is the range of a norm one projection in $X^{* *}$ and if $X$ is an ideal in $Y$ (that is $H B(X, Y) \neq \emptyset$ ), then there exist $\phi \in H B(X, Y)$ and $\Phi \in H B(\mathcal{A}(Z, X), \mathcal{A}(Z, Y))$ such that $Z=Z_{\Phi \phi}$.

Proof. Let $\psi \in H B(X, Y)$ and let $P: X^{* *} \rightarrow X$ be a norm one projection onto $X$. Define $\Phi: \mathcal{A}(Z, X)^{*} \rightarrow \mathcal{A}(Z, Y)^{*}$ by

$$
(\Phi F)(T)=F\left(P \circ \psi_{\mid Y}^{*} \circ T\right), \quad F \in \mathcal{A}(Z, X)^{*}, \quad T \in \mathcal{A}(Z, Y) .
$$

Then $\Phi$ is linear and $\|\Phi F\| \leq\|F\|$. Since $\psi^{*} x=x$ for all $x$, that is $\psi_{\mid X}^{*}=I_{X}$, we have, for all $S \in \mathcal{A}(Z, X)$,

$$
(\Phi F)(S)=F\left(P \circ I_{X} \circ S\right)=F(S),
$$

meaning that $\Phi F$ is an extension of $F$. Hence $\Phi$ is a Hahn-Banach extension operator.

Moreover, if $x^{*} \in X^{*}, T \in \mathcal{A}(Z, Y)$, and $z \in Z$, then

$$
\begin{aligned}
\left(\Phi\left(x^{*} \otimes z\right)\right)(T) & =\left(x^{*} \otimes z\right)\left(\left.P \circ \psi^{*}\right|_{Y} \circ T\right)=x^{*}\left(\left.P \psi^{*}\right|_{Y} T z\right) \\
& =\left(\left(\psi_{\mid Y}^{*}\right)^{*} P^{*} x^{*}\right)(T z)=\left(\phi x^{*}\right)(T z),
\end{aligned}
$$

where $\phi=\left(\psi_{\mid Y}^{*}\right)^{*} \circ P^{*}$. Noting that $\phi \in H B(X, Y)$, we have $Z=Z_{\Phi \phi}$ as desired.

Let us recall that $X$ is always an ideal in $X^{* *}$ because the canonical embedding $J_{X^{*}}: X^{*} \rightarrow X^{* * *}$ is a Hahn-Banach extension operator. But if we have a norm one projection $P$ from $X^{* *}$ onto $X$ as in Proposition 2.1, then clearly $P^{*} \in H B\left(X, X^{* *}\right)$.

Corollary 2.2. Let $X$ and $Z$ be Banach spaces and let $\mathcal{A}$ be a closed operator ideal. If $X$ is the range of a norm one projection $P$ in $X^{* *}$, then $P^{*} \in H B\left(X, X^{* *}\right)$ and there exist $\Phi \in H B\left(\mathcal{A}(Z, X), \mathcal{A}\left(Z, X^{* *}\right)\right)$ such that $Z=Z_{\Phi P^{*}}$.

Proof. It suffices to note that if we put $\psi=P^{*}$, then $\psi_{\mid X^{* *}}^{*}=P$ and the proof of Proposition 2.1 will yield $\Phi \in H B\left(\mathcal{A}(Z, X), \mathcal{A}\left(Z, X^{* *}\right)\right)$ defined by

$$
(\Phi F)(T)=F(P \circ T), \quad F \in \mathcal{A}(Z, X)^{*}, \quad T \in \mathcal{A}\left(Z, X^{* *}\right),
$$

and also the equality $Z=Z_{\Phi P^{*}}$.

The following theorem is the main result of this paper. Its proof relies on Theorem 1.3 , 
Theorem 2.3. Let $X$ be a closed subspace of a Banach space $Y$ and let $Z$ be a Banach space having the Radon-Nikodým property. Let $\mathcal{A}$ and $\mathcal{B}$ be closed operator ideals satisfying $\mathcal{A} \subseteq \mathcal{B}$. If $\mathcal{A}(\hat{Z}, X)$ is an ideal in $\mathcal{B}(\hat{Z}, Y)$ for all equivalently renormed versions $\hat{Z}$ of $Z$, then there exist

$$
\phi \in H B(X, Y) \quad \text { and } \quad \Phi \in H B(\mathcal{A}(Z, X), \mathcal{B}(Z, Y))
$$

such that $Z=Z_{\Phi \phi}$. Moreover, the (non-empty) subset

$$
\mathcal{F}_{Z}=\left\{\phi \in H B(X, Y): \exists \Phi \in H B(\mathcal{A}(Z, X), \mathcal{B}(Z, Y)) \text { such that } Z=Z_{\Phi \phi}\right\}
$$

of $\mathcal{L}\left(X^{*}, Y^{*}\right)=\left(Y \hat{\otimes}_{\pi} X^{*}\right)^{*}$ is weak compact. $^{*}$

Proof. Let $\Phi \in H B(\mathcal{A}(Z, X), \mathcal{B}(Z, Y))$. By Theorem 1.3, there exists $\left\{\phi_{i}: i \in\right.$ $I\} \subseteq H B(X, Y)$ such that

$$
Z=\sum_{i \in I} \oplus_{1} Z_{\Phi \phi_{i}}, \quad Z_{\Phi \phi_{i}} \neq\{0\}, \quad i \in I
$$

If $I$ consists of only one element, then we have nothing to prove. If $I$ contains more than just one element, then denoting $W=Z_{\Phi \phi_{i}}$ and $V=\sum_{j \in I \backslash\{i\}} \oplus_{1} Z_{\Phi \phi_{j}}$ for some fixed $i \in I$, we can write $Z=W \oplus_{1} V$.

For every $\epsilon \in(0,1]$, let $Z_{\epsilon}=\left(Z,\|\cdot\|_{\epsilon}\right)$ be the equivalently renormed version of $Z$ with $\|z\|_{\epsilon}=\left(\|w\|^{1+\epsilon}+\|v\|^{1+\epsilon}\right)^{1 / 1+\epsilon}, w \in W, v \in V$, and let $\Phi_{\epsilon} \in$ $H B\left(\mathcal{A}\left(Z_{\epsilon}, X\right), \mathcal{B}\left(Z_{\epsilon}, Y\right)\right)$. By a theorem due to Behrends (see [1, 22, p. 8], or [3]), a Banach space (except $\left(\mathbb{R}^{2},\|\cdot\|_{\infty}\right)$ which does not contain any nontrivial $L^{p_{-}}$ summand for $1<p<\infty)$ cannot contain nontrivial $L^{p}$-summands and nontrivial $L^{r}$-summands simultanously if $p \neq r$. Since $Z_{\epsilon}$ contains nontrivial $L^{1+\epsilon}$-summands ( $W$ and $V$ ), it does not contain any nontrivial $L^{1}$-summand. Therefore, by Theorem 1.3, there exists $\phi_{\epsilon} \in H B(X, Y)$ such that $Z_{\epsilon}=\left(Z_{\epsilon}\right)_{\Phi_{\epsilon} \phi_{\epsilon}}$.

Let us denote by $I_{\epsilon}: Z_{\epsilon} \rightarrow Z$ the identity mapping. Then $\left\|I_{\epsilon}^{-1}\right\|=1$ and $\left\|I_{\epsilon}\right\| \rightarrow 1$ as $\epsilon \rightarrow 0$. Define $\Psi_{\epsilon} \in \mathcal{L}\left(\mathcal{A}(Z, X)^{*}, \mathcal{B}(Z, Y)^{*}\right)$ by

$$
\left(\Psi_{\epsilon} F\right)(T)=\left(\Phi_{\epsilon} F_{\epsilon}\right)\left(T \circ I_{\epsilon}\right), \quad F \in \mathcal{A}(Z, X)^{*}, \quad T \in \mathcal{B}(Z, Y),
$$

where $F_{\epsilon} \in \mathcal{A}\left(Z_{\epsilon}, X\right)^{*}$ is defined by

$$
F_{\epsilon}(S)=F\left(S \circ I_{\epsilon}^{-1}\right), \quad S \in \mathcal{A}\left(Z_{\epsilon}, X\right) .
$$

Then $\left\|F_{\epsilon}\right\| \leq\|F\|$ and $\left\|\Psi_{\epsilon}\right\| \leq\left\|I_{\epsilon}\right\| \leq \sqrt{2}$ for $\epsilon \in(0,1]$. Since $\left(\Psi_{\epsilon}\right)_{\epsilon \in(0,1]}$ is a bounded net in $\mathcal{L}\left(\mathcal{A}(Z, X)^{*}, \mathcal{B}(Z, Y)^{*}\right)=\left(\mathcal{B}(Z, Y) \hat{\otimes}_{\pi} \mathcal{A}(Z, X)^{*}\right)^{*}$, it has a subnet $\left(\Psi_{\epsilon(\nu)}\right)$ that converges weak* to some $\Psi \in \mathcal{L}\left(\mathcal{A}(Z, X)^{*}, \mathcal{B}(Z, Y)^{*}\right)$. Noting that $\phi_{\epsilon(\nu)} \in B_{\mathcal{L}\left(X^{*}, Y^{*}\right)}=B_{\left(Y \hat{\otimes}_{\pi} X^{*}\right)^{*}}$ and by passing to a subnet, we may assume that $\left(\phi_{\epsilon(\nu)}\right)$ converges weak ${ }^{*}$ to some $\phi \in B_{\mathcal{L}\left(X^{*}, Y^{*}\right)}$. It is clear that $\phi \in H B(X, Y)$ because $\phi_{\epsilon(\nu)} \in H B(X, Y)$ and

$$
\left(\phi x^{*}\right)(y)=\phi\left(y \otimes x^{*}\right)=\lim _{\nu} \phi_{\epsilon(\nu)}\left(y \otimes x^{*}\right)=\lim _{\nu}\left(\phi_{\epsilon(\nu)} x^{*}\right)(y)
$$

for all $x^{*} \in X^{*}$ and $y \in Y$. It remains to show that $\Psi \in H B(\mathcal{A}(Z, X), \mathcal{B}(Z, Y))$ and $Z=Z_{\Psi \phi}$.

Consider any $F \in \mathcal{A}(Z, X)^{*}$. Since, for all $T \in \mathcal{B}(Z, Y)$,

$$
(\Psi F)(T)=\Psi(T \otimes F)=\lim _{\nu} \Psi_{\epsilon(\nu)}(T \otimes F)=\lim _{\nu}\left(\Psi_{\epsilon(\nu)} F\right)(T),
$$

we get that

$$
\|\Psi F\| \leq \limsup _{\nu}\left\|\Psi_{\epsilon(\nu)}\right\|\|F\| \leq \lim _{\nu}\left\|I_{\epsilon(\nu)}\right\|\|F\|=\|F\| .
$$


Since for all $S \in \mathcal{A}(Z, X)$ we also have

$$
\begin{aligned}
(\Psi F)(S) & =\lim _{\nu}\left(\Phi_{\epsilon(\nu)} F_{\epsilon(\nu)}\right)\left(S \circ I_{\epsilon(\nu)}\right) \\
& =\lim _{\nu} F_{\epsilon(\nu)}\left(S \circ I_{\epsilon(\nu)}\right)=\lim _{\nu} F\left(S \circ I_{\epsilon(\nu)} \circ I_{\epsilon(\nu)}^{-1}\right)=F(S),
\end{aligned}
$$

we conclude that $\Psi \in H B(\mathcal{A}(Z, X), \mathcal{B}(Z, Y))$.

Finally, for arbitrary $x^{*} \in X^{*}, T \in \mathcal{B}(Z, Y)$, and $z \in Z$, we have

$$
\begin{aligned}
\left(\Psi\left(x^{*} \otimes z\right)\right)(T) & =\lim _{\nu}\left(\Phi_{\epsilon(\nu)}\left(x^{*} \otimes I_{\epsilon(\nu)}^{-1} z\right)\right)\left(T \circ I_{\epsilon(\nu)}\right) \\
& =\lim _{\nu}\left(\phi_{\epsilon(\nu)} x^{*}\right)\left(T I_{\epsilon(\nu)} I_{\epsilon(\nu)}^{-1} z\right) \\
& =\lim _{\nu}\left(\phi_{\epsilon(\nu)} x^{*}\right)(T z)=\left(\phi x^{*}\right)(T z),
\end{aligned}
$$

meaning that $Z=Z_{\Psi \phi}$.

For the "moreover" part, let us note that the above proof shows, in particular, that $H B(X, Y)$ is a weak ${ }^{*}$ compact subset of $\mathcal{L}\left(X^{*}, Y^{*}\right)=\left(Y \hat{\otimes}_{\pi} X^{*}\right)^{*}$. Therefore if we consider a net $\left(\phi_{\alpha}\right)$ in $\mathcal{F}_{Z}$ together with a net $\left(\Phi_{\alpha}\right)$ in $H B(\mathcal{A}(Z, X), \mathcal{B}(Z, Y))$ satisfying $Z=Z_{\Phi_{\alpha} \phi_{\alpha}}$ for all $\alpha$, then by passing to a subnet, we may assume that $\phi_{\alpha} \rightarrow \phi \in H B(X, Y)$ and $\Phi_{\alpha} \rightarrow \Phi \in H B(\mathcal{A}(Z, X), \mathcal{B}(Z, Y))$ with respect to the corresponding weak* topologies. We also have that $Z=Z_{\Phi \phi}$ because

$$
\begin{aligned}
\left(\Phi\left(x^{*} \otimes z\right)\right)(T) & =\lim _{\alpha}\left(\Phi_{\alpha}\left(x^{*} \otimes z\right)\right)(T) \\
& =\lim _{\alpha}\left(\phi_{\alpha} x^{*}\right)(T z)=\left(\phi x^{*}\right)(T z)
\end{aligned}
$$

for all $x^{*} \in X^{*}, T \in \mathcal{B}(Z, Y)$, and $z \in Z$.

Theorem 2.4. Let $X$ be a closed subspace of a Banach space $Y$. Let $\mathcal{A}$ and $\mathcal{B}$ be closed operator ideals satisfying $\mathcal{A} \subseteq \mathcal{B}$. If $\mathcal{A}(W, X)$ is an ideal in $\mathcal{B}(W, Y)$ for all Banach spaces $W$ having the Radon-Nikodym property, then there exists $\phi \in H B(X, Y)$ such that, for every Banach space $Z$ having the Radon-Nikodym property, there exists $\Phi \in H B(\mathcal{A}(Z, X), \mathcal{B}(Z, Y))$ such that $Z=Z_{\Phi \phi}$.

Proof. Let $\mathcal{Z}$ be the collection of all Banach spaces $Z$ having the Radon-Nikodým property. We need to show that $\bigcap_{Z \in \mathcal{Z}} \mathcal{F}_{Z}$ is non-empty. Since $B_{\mathcal{L}\left(X^{*}, Y^{*}\right)}=$ $B_{\left(Y \hat{\otimes}_{\pi} X^{*}\right)^{*}}$ is compact with respect to the weak* topology and, by Theorem 2.3 its subsets $\mathcal{F}_{Z}, Z \in \mathcal{Z}$, are closed, the claim follows whenever each finite subfamily of $\left\{\mathcal{F}_{Z}: Z \in \mathcal{Z}\right\}$ has non-empty intersection.

Consider $Z_{1}, \ldots, Z_{n} \in \mathcal{Z}$ and denote $Z=\sum_{i=1}^{n} \oplus_{1} Z_{i}$. Let $I_{i}: Z_{i} \rightarrow Z$ and $P_{i}$ : $Z \rightarrow Z_{i}$ be the natural embedding and projection. Since $Z \in \mathcal{Z}$, by Theorem 2.3 there exist $\phi \in H B(X, Y)$ and $\Phi \in H B(\mathcal{A}(Z, X), \mathcal{B}(Z, Y))$ such that $Z=Z_{\Phi \phi}$. We shall prove that

$$
\phi \in \bigcap_{i=1}^{n} \mathcal{F}_{Z_{i}} .
$$

For this, let us fix $i \in\{1, \ldots, n\}$ and define $\Psi \in \mathcal{L}\left(\mathcal{A}\left(Z_{i}, X\right)^{*}, \mathcal{B}\left(Z_{i}, Y\right)^{*}\right)$ by

$$
(\Psi F)(T)=(\Phi G)\left(T \circ P_{i}\right), \quad F \in \mathcal{A}\left(Z_{i}, X\right)^{*}, \quad T \in \mathcal{B}\left(Z_{i}, Y\right),
$$

where $G \in \mathcal{A}(Z, X)^{*}$ is defined by

$$
G(S)=F\left(S \circ I_{i}\right), \quad S \in \mathcal{A}(Z, X) .
$$


Then

$$
\|\Psi F\| \leq\|\Phi G\|=\|G\| \leq\|F\|
$$

and

$$
(\Psi F)(T)=(\Phi G)\left(T \circ P_{i}\right)=G\left(T \circ P_{i}\right)=F\left(T \circ P_{i} \circ I_{i}\right)=F(T)
$$

for all $F \in \mathcal{A}\left(Z_{i}, X\right)^{*}$ and $T \in \mathcal{A}\left(Z_{i}, X\right)$. Hence $\Psi \in H B\left(\mathcal{A}\left(Z_{i}, X\right), \mathcal{B}\left(Z_{i}, Y\right)\right)$. Moreover, for all $x^{*} \in X^{*}, T \in \mathcal{B}\left(Z_{i}, Y\right)$, and $w \in Z_{i}$, we have (since $Z=Z_{\Phi \phi}$ )

$$
\begin{aligned}
\left(\Psi\left(x^{*} \otimes w\right)\right)(T) & =\left(\Phi\left(x^{*} \otimes I_{i} w\right)\right)\left(T \circ P_{i}\right) \\
& =\left(\phi x^{*}\right)\left(T P_{i} I_{i} w\right) \\
& =\left(\phi x^{*}\right)(T w),
\end{aligned}
$$

meaning that $Z_{i}=\left(Z_{i}\right)_{\Psi \phi}$. Consequently, $\phi \in \mathcal{F}_{Z_{i}}$.

We conclude with some applications of Theorem 2.4. They also use results from the recent paper [9] by the authors.

Corollary 2.5. Let $X$ be an ideal in a Banach space $Y$. Then there exists $\phi \in$ $H B(X, Y)$ such that, for every Banach space $Z$ having the Radon-Nikodym property, there exists $\Phi \in H B(\overline{\mathcal{F}(Z, X)}, \overline{\mathcal{F}(Z, Y)})$ such that $Z=Z_{\Phi \phi}$.

Proof. This is an immediate consequence of Theorem 2.4 because $\overline{\mathcal{F}(W, X)}$ is an ideal in $\overline{\mathcal{F}(W, Y)}$ for all Banach spaces $W$ whenever $X$ is an ideal in $Y$ (see [9] Corollary 2.3]).

Corollary 2.6. Let $X$ be a closed subspace of a Banach space $Y$. If $\mathcal{K}(W, X)$ is an ideal in $\mathcal{K}(W, Y)$ for all separable reflexive Banach spaces $W$, then there exists $\phi \in H B(X, Y)$ such that, for every Banach space $Z$ having the Radon-Nikodym property, there exists $\Phi \in H B(\mathcal{K}(Z, X), \mathcal{K}(Z, Y))$ such that $Z=Z_{\Phi \phi}$.

Proof. By [9, Theorem 4.2], if $\mathcal{K}(W, X)$ is an ideal in $\mathcal{K}(W, Y)$ for all separable reflexive $W$, then it is also an ideal for all Banach spaces $W$. And Theorem 2.4 applies.

Corollary 2.7. Let $X$ be a closed subspace of a Banach space $Y$. If $\mathcal{K}(W, X)$ is an ideal in $\mathcal{W}(W, Y)$ for all reflexive Banach spaces $W$, then there exists $\phi \in H B(X, Y)$ such that, for every Banach space $Z$ having the Radon-Nikodym property, there exists $\Phi \in H B(\mathcal{K}(Z, X), \mathcal{W}(Z, Y))$ such that $Z=Z_{\Phi \phi}$.

Proof. In this case, by [9, Theorem 4.4], $\mathcal{K}(W, X)$ is an ideal in $\mathcal{W}(W, Y)$ for all Banach spaces $W$, and Theorem 2.4 applies.

Corollary 2.8. Let $X$ be an ideal in a Banach space $Y$. If $X^{*}$ has the compact approximation property with conjugate operators, then there exists $\phi \in H B(X, Y)$ such that, for every Banach space $Z$ having the Radon-Nikodym property, there exists $\Phi \in H B(\mathcal{K}(Z, X), \mathcal{W}(Z, Y))$ such that $Z=Z_{\Phi \phi}$.

Proof. In this case, again, Theorem 2.4 applies because $\mathcal{K}(W, X)$ is an ideal in $\mathcal{W}(W, Y)$ for all Banach spaces $W$ (see [9, Corollary 4.7]).

In fact (through [9] Corollary 4.7]), Corollary [2.8 is also an application of Theorem 4.6 of [9]. Let us compare these two results. Their assumptions concerning $X$ and $Y$ are the same. Corollary 2.8 applies to $Z$ having the Radon-Nikodým property, whereas in Theorem 4.6 of [9], $Z$ is supposed to be reflexive. On the 
other hand, Theorem 4.6 of [9] claims more: for any $\phi \in H B(X, Y)$, there exists $\Phi \in H B(\mathcal{K}(Z, X), \mathcal{W}(Z, Y))$ such that $Z=Z_{\Phi \phi}$.

\section{REFERENCES}

[1] E. Behrends. L ${ }^{p}$-Struktur in Banachräumen. Studia Math. 55 (1976) 71-85. MR 53:6286

[2] E. Behrends, R. Danckwerts, R. Evans, S. Göbel, P. Greim, K. Meyfarth, and W. MüLLER. L $L^{p}$-Structure in Real Banach Spaces. Lecture Notes in Mathematics 613, Springer-Verlag, Berlin-Heidelberg-New York (1977). MR 58:30082

[3] M. Cambern, K. Jarosz, And G. Wodinski. Almost- $L^{p}$-projections and $L^{p}$ isomorphisms. Proc. Royal Soc. Edinburgh, Ser. A 113 (1989) 13-25. MR 91b:46014

[4] J.A. Clarkson. Uniformly convex spaces. Trans. Amer. Math. Soc. 40 (1936) 396-414.

[5] J. Diestel. Geometry of Banach Spaces - Selected Topics. Lecture Notes in Mathematics 485, Springer-Verlag, Berlin-Heidelberg-New York (1975). MR 57:1079

[6] J. Diestel And J.J. Uhl, JR. Vector Measures. Mathematical Surveys 15, Amer. Math. Soc., Providence, Rhode Island (1977). MR 56:12216

[7] G. Godefroy, N.J. Kalton, and P.D. Saphar. Unconditional ideals in Banach spaces. Studia Math. 104 (1993) 13-59. MR 94k:46024

[8] R.C. James. Uniformly non-square Banach spaces. Ann. of Math. 80 (1964) 542-550. MR 30:4139

[9] Å. Lima And E. OJa. Ideals of compact operators. (submitted).

[10] J. Lindenstrauss and L. Tzafriri. Classical Banach Spaces I. Ergebnisse der Mathematik und ihrer Grenzgebiete 92, Springer-Verlag, Berlin-Heidelberg-New York (1977). MR 58:17766

[11] A. Pietsch. Operator Ideals. North-Holland Publishing Company, Amsterdam-New YorkOxford (1980). MR 81j:47001

Department of Mathematics, Agder College, Gimlemoen 257, Serviceboks 422, 4604 KRISTIANSAND, NORWAY

E-mail address: Asvald.Lima@ia.no

Faculty of Mathematics, Tartu University, Vanemuise 46, EE-51014 Tartu, Estonia

E-mail address: eveoja@math.ut.ee 